Submitted to the Astrophysical Journal

Preprint typeset using $\mathrm{LT}_{\mathrm{E}} \mathrm{X}$ style emulateapj v. 05/12/14

\title{
SONGLINES FROM DIRECT COLLAPSE SEED BLACK HOLES: EFFECTS OF X-RAYS ON BLACK HOLE GROWTH AND STELLAR POPULATIONS
}

\author{
Aycin Aykutalp ${ }^{1,2}$, John H. Wise ${ }^{1}$, MARCo SPAAns $^{3}$, And Rowin MEIJERINK ${ }^{4}$ \\ ${ }^{1}$ Center for Relativistic Astrophysics, School of Physics, Georgia Institute of Technology, 837 State Street, Atlanta, GA 30332; \\ aycin.aykutalp@physics.gatech.edu \\ ${ }^{2}$ Scuola Normale Superiore, Piazza dei Cavalieri 7, I-56126 Pisa, Italy \\ ${ }^{3}$ Kapteyn Astronomical Institute, University of Groningen, PO Box 800, 9700 AV Groningen, The Netherlands and \\ ${ }^{4}$ Leiden Observatory, Leiden University, P.O. Box 9513, NL-2300 RA Leiden, The Netherlands \\ Submitted 2014 August 29; accepted 2014 October 7
}

\begin{abstract}
In the last decade, the growth of supermassive black holes (SMBHs) has been intricately linked to galaxy formation and evolution and is a key ingredient in the assembly of galaxies. To investigate the origin of SMBHs, we perform cosmological simulations that target the direct collapse black hole (DCBH) seed formation scenario in the presence of two different strong Lyman-Werner (LW) background fields. These simulations include the X-ray irradiation from a central massive black hole $(\mathrm{MBH}), \mathrm{H}_{2}$ self-shielding and stellar feedback from metalfree and metal-enriched stars. We find in both simulations that local X-ray feedback induces metal-free star formation $\sim 0.5 \mathrm{Myr}$ after the $\mathrm{MBH}$ forms. The $\mathrm{MBH}$ accretion rate reaches a maximum of $10^{-3} M_{\odot} \mathrm{yr}^{-1}$ in both simulations. However, the duty cycle differs which is derived to be $6 \%$ and $50 \%$ for high and low LW cases, respectively. The MBH in the high LW case grows only $\sim 6 \%$ in 100 Myr compared to $16 \%$ in the low LW case. We find that the maximum accretion rate is determined by the local gas thermodynamics whereas the duty cycle is determined by the large scale gas dynamics and gas reservoir. We conclude that radiative feedback from the central $\mathrm{MBH}$ plays an important role in star formation in the nuclear regions and stifling initial $\mathrm{MBH}$ growth, relative to the typical Eddington rate argument, and that initial $\mathrm{MBH}$ growth might be affected by the local LW radiation field.

Keywords: galaxies: active - galaxies: formation - galaxies: nuclei
\end{abstract}

\section{INTRODUCTION}

Observations of high redshift $(z>6)$ quasars suggest that these objects are powered by SMBHs with masses on the order of $10^{9} M_{\odot}$ (Fan et al. 2003, 2006; Kurk et al. 2007; Mortlock et al. 2011). Fundamental to understanding their existence within the first billion years after the Big Bang, is the identification of their formation processes, growth rate and evolution through cosmic time. There are three plausible scenarios for the formation of these seed black holes: a) they are the remnants of the first stars $\left(M_{\mathrm{BH}} \sim 10^{2} M_{\odot}\right.$, Volonteri et al. 2003; Volonteri \& Rees 2005; Johnson \& Bromm 2007), b) they formed in dense star clusters through mergers $\left(M_{\mathrm{BH}} \sim 10^{4} M_{\odot}\right.$, Begelman \& Rees 1978; Ebisuzaki et al. 2001), and c) they formed in the isothermal direct gaseous collapse in atomic cooling halos $\left(M_{\mathrm{BH}} \sim 10^{4}-10^{6} M_{\odot}\right.$, Haehnelt \& Rees 1993; Umemura et al. 1993; Bromm \& Loeb 2003; Spaans \& Silk 2006; Wise et al. 2008) in pre-galactic objects.

In this work we simulate the latter scenario for the formation of a $\mathrm{MBH}\left(M_{\mathrm{MBH}} \sim 10^{4}-10^{6} M_{\odot}\right)$. In order to form a $\mathrm{MBH}$ through direct collapse, fragmentation into stars needs to be prevented. Here, it is important that the gas cloud has primordial composition. Metal-enriched gas cools very efficiently by fine-structure metal lines to temperatures of $T<100 \mathrm{~K}$, causing gas cloud to fragment into smaller clumps of $\leq 100 M_{\odot}$, since the Jeans mass $\left(M_{\mathrm{J}}\right)$ scales with the temperature of the ambient gas as $M_{\mathrm{J}} \propto T^{3 / 2}$. In metalfree gas the dominant coolant below $10^{4} \mathrm{~K}$ is molecular hydrogen, $\mathrm{H}_{2}$, which can cool the gas down to $200 \mathrm{~K}$ through rovibrational transitions. Thus, to avoid fragmentation the formation of $\mathrm{H}_{2}$ needs to be suppressed. Indeed, in their studies
Bromm \& Loeb (2003) and Spaans \& Silk (2006) have shown that in the absence of $\mathrm{H}_{2}$ fragmentation is sufficiently prevented.

$\mathrm{H}_{2}$ is fragile and can easily be dissociated by photons in the LW bands $(\mathrm{E}=11.2-13.6 \mathrm{eV})$. Recent works on the photodissociation of $\mathrm{H}_{2}$ in protogalaxies (Shang et al. 2010; Wolcott-Green et al. 2011; Latif et al. 2014a,b) have shown that a LW flux in the range of $10^{2}-10^{5} J_{21}$, where $J_{21}$ is the specific intensity just below $13.6 \mathrm{eV}\left(J_{21}=10^{-21} \mathrm{erg} \mathrm{s}^{-1}\right.$ $\left.\mathrm{cm}^{-2} \mathrm{sr}^{-1} \mathrm{~Hz}^{-1}\right)$, is sufficient to prevent $\mathrm{H}_{2}$ formation, and hence fragmentation, in halos with $T_{\mathrm{vir}}=10^{4}-10^{5} \mathrm{~K}$. Moreover, Inayoshi \& Omukai (2012) and Visbal et al. (2014) have argued that, there is a zone of no-return for a collapsing gas cloud to form a DCBH without fragmenting into smaller clumps, which depends on the density $\left(n>10^{4} \mathrm{~cm}^{-3}\right)$ and temperature $\left(T \geq 10^{4} \mathrm{~K}\right)$ of the collapsing gas. The latter authors also emphasized that delaying $\mathrm{H}_{2}$ cooling in a collapsing gas clouds, in the absence of a LW background radiation, will not be enough to avoid fragmentation, since throughout the collapse the density of the cloud will increase and $\mathrm{H}_{2}$ cooling eventually take over.

After the seed black hole forms, it mainly grows through gas accretion, where the gas reservoir depends on mergers and/or galaxy interactions. The accretion of gas onto the central black hole yields a luminous radiation source with a broad spectrum. As studied in Aykutalp et al. (2013) (hereafter Paper I), the thermodynamics of the gas in the inner region of an active galactic nucleus (AGN) is dominated by the X-ray radiation produced by the infall of gas onto the central $\mathrm{MBH}$. Thus, to understand the growth of $\mathrm{MBH}$ it is crucial to include the X-ray radiation from the AGN. In order to incorporate the effects of X-rays on the $\mathrm{MBH}$ growth and on star formation 
around an AGN, in a self-consistent manner, we have implemented the XDR chemical network of Meijerink \& Spaans (2005) into Enzo (for details, see Paper I).

The aims of this paper are, for the DCBH formation scenario, (1) to investigate the possible connection between SMBHs and the formation and evolution of atomic cooling halos, (2) to follow the accretion history of the seed MBH, and (3) to assess the effects of episodic X-ray irradiation on the ambient gas and hence, on the growth of the seed MBH. This paper is structured as follows. In Section 2, we describe our simulation setup. We present our results and implications in Section 3. Finally, in Section 4, we discuss our findings and possible caveats in our simulations.

\section{SIMULATIONS}

In this work, we use the Eulerian adaptive mesh refinement (AMR) hydrodynamic code Enzo (Bryan et al. 2014), version 2.1, which is modified to include metallicity dependent XDR physics and $\mathrm{H}_{2}$ self-shielding. We perform simulations in a three-dimensional periodic box with a side length of $3 \mathrm{~h}^{-1}$ Mpc, initialized at $z=99$ by using inits, a package that uses Zel'dovich approximation and is included with the Enzo distribution. The size of the root grid is $128^{3}$ with three nested subgrids, each refined by a factor of two. The finest grid has an effective resolution of $1024^{3}$ with a side length of $375 \mathrm{~h}^{-1}$ kpc. Refinement is restricted to the finest grid and occurs on baryon overdensities of $3 \times 2^{-0.2 l}$. Here 1 is the AMR level, and the negative exponent means that the mass resolution in the calculations is super-Lagrangian (O'Shea \& Norman 2008). The maximum level of refinement that is reached in the finest grid is 10 , allowing us to have a resolution of 3.6 proper pc. The virial mass of the most massive halo at redshift $z=15$ is $M_{v i r}=2.2 \times 10^{8} M_{\odot}$, where $M_{v i r}$ is the mass in a sphere that encloses an average dark matter overdensity of 200.

We use Wilkinson Microwave Anisotropy Probe seven-year cosmological parameters (Komatsu et al. 2009): $\Omega_{\Lambda}=0.734$, $\Omega_{m}=0.266, \Omega_{b}=0.0449, \sigma_{8}=0.81$, and $h=0.701$. Here, $\Omega_{\Lambda}$ is the vacuum energy, $\Omega_{m}$ is the matter density, $\Omega_{b}$ is the baryon density, $\sigma_{8}$ is the variance of random mass fluctuations in a sphere of radius $8 h^{-1} \mathrm{Mpc}$, and $h$ is the Hubble parameter in units of $100 \mathrm{~km} \mathrm{~s}^{-1} \mathrm{Mpc}^{-1}$.

\section{1. $\mathrm{H}_{2}$ Self-Shielding \& Black Hole Accretion}

For a $\mathrm{MBH}$ to form at $z=15$ in the direct collapse scenario, its birth cloud cannot fragment through $\mathrm{H}_{2}$ cooling as it collapses. Therefore, we introduce a strong LW background radiation field that could originate from a nearby galaxy (Dijkstra et al. 2008, 2014; Agarwal et al. 2012, 2013). We perform two simulations, one with a $\mathrm{LW}$ background intensity of $10^{3} J_{21}$ (hereafter $B G_{3}$ ) and one for $10^{5} J_{21}$ (hereafter $B G_{5}$ ). We turn on the LW background at $z=30$ and keep it on for the remainder of the simulations. Recent works by Wolcott-Green et al. (2011) and Regan et al. (2014) have shown that such $J_{\text {LW }}$ values are sufficient to keep the halo almost $\mathrm{H}_{2}$ free.

$\mathrm{H}_{2}$ self-shielding is crucial for the formation of stars in regions where the $\mathrm{H}_{2}$ column densities exceed $10^{14} \mathrm{~cm}^{-2}$. Thus, we take into account $\mathrm{H}_{2}$ self-shielding as well as the attenuation of the $\mathrm{MBH}$ radiation from $\mathrm{H} \mathrm{I}, \mathrm{He}$ I, and $\mathrm{He}$ II. For $\mathrm{H}_{2}$ self-shielding, we use a local approximation and multiply the
$\mathrm{H}_{2}$ photo-dissociation rate by a self-shielding factor $f_{\text {sh }}$

$$
\begin{aligned}
f_{\mathrm{sh}} & =\min \left[1,\left(\frac{\mathrm{N}_{\mathrm{H}_{2}}}{10^{14} \mathrm{~cm}^{-2}}\right)^{-3 / 4}\right], \\
N_{\mathrm{H}_{2}} & =f_{\mathrm{H}_{2}} n_{\text {tot }} \lambda_{\mathrm{J}}
\end{aligned}
$$

to correct the impinging UV flux in the LW band (Draine \& Bertoldi 1996; Shang et al. 2010). Here, $N_{\mathrm{H}_{2}}$ is the $\mathrm{H}_{2}$ column density, $n_{\text {tot }}$ is the total particle number density, and $\lambda_{\mathrm{J}}$ is the jeans length $\left(\lambda_{\mathrm{J}}=\sqrt{\frac{15 k_{b} T}{4 \pi G \mu \rho}}\right)$. We note that in their work, Wolcott-Green et al. (2011) have shown that using the $\lambda_{\mathrm{J}}$ causes underestimating the $N_{\mathrm{H} 2}$ about an order of magnitude in the low density regimes $\left(n<10^{4} \mathrm{~cm}^{-3}\right)$, and Sobolev length (Sobolev 1957), which takes into account the velocity gradients in the gas, is a more accurate method (see the discussion section for further details).

At redshift $z=15$ we insert a MBH with a mass of $M=5 \times$ $10^{4} M_{\odot}$. We include the radiative feedback from the MBH using the Enzo radiation transport module Moray (Wise \& Abel 2011). The radiation from the $\mathrm{MBH}$ is modeled as follows. To calculate the gas temperature, we use an XDR grid of models produced for a large parameter space in X-ray flux $F_{\mathrm{X}}$, number density $n$, column density $N_{\mathrm{H}}$, and metallicity $Z / Z_{\odot}$ (see section 2.3). We employ Moray to compute the full (chemical, thermal and hydrodynamic) response of X-ray exposed gas. We use an $N_{\mathrm{HI}}$ lookup-table for a polychromatic $\mathrm{X}$-ray spectrum to calculate the attenuation in each line of sight (Mellema et al. 2006). The radiative transfer equation is numerically solved before the simulation, giving a relative ionizing photon flux $\mathrm{I}_{\nu}$ as a function of the neutral hydrogen column density $N_{\mathrm{H}}$. The relative ionizing photon flux for $\mathrm{HI}$, HeI, and HeII is computed and stored for 300 column densities, equally log-spaced over the range $N_{\mathrm{H}}=10^{12}-10^{25} \mathrm{~cm}^{-2}$. The details of this approach are described in Paper I.

For the accretion of gas onto the $\mathrm{MBH}$, we use the prescription of Kim et al. (2011). We calculate the accretion rate by using the Eddington-limited spherical Bondi-Hoyle equation

$$
\begin{aligned}
\dot{M}_{\mathrm{BH}} & =\min \left(\dot{\mathrm{M}}_{\mathrm{B}}, \dot{\mathrm{M}}_{\mathrm{Edd}}\right) \\
& =\min \left(\frac{4 \pi \mathrm{G}^{2} \mathrm{M}_{\mathrm{BH}}^{2} \rho_{\mathrm{B}}}{\mathrm{c}_{\mathrm{s}}^{3}}, \frac{4 \pi \mathrm{GM}_{\mathrm{BH}} \mathrm{m}_{\mathrm{p}}}{\epsilon \sigma_{\mathrm{T}} \mathrm{c}}\right),
\end{aligned}
$$

where $\mathrm{G}$ is the gravitational constant, $M_{\mathrm{BH}}$ is the mass of the $\mathrm{MBH}, \rho_{\mathrm{B}}$ is the density at the Bondi radius, $\mathrm{c}_{s}$ is the sound speed, $m_{\mathrm{p}}$ is the mass of a proton, $\epsilon$ is the radiative efficiency, and $\sigma_{\mathrm{T}}$ is the Thomson scattering cross-section. And the Bondi radius

$$
R_{\mathrm{B}}=\frac{2 G M_{\mathrm{BH}}}{c_{\mathrm{S}}^{2}} .
$$

The gas inside a Bondi radius is accreted onto the $\mathrm{MBH}$ and it is uniformly subtracted from grid cells.

\subsection{Star Formation \& Feedback}

In order to model the interplay between stellar and black hole feedback, we employ different recipes for Pop III and PopII/I star formation. We allow star formation to occur only in the finest AMR levels. In our simulation a Pop III star particle, representing a single star, is created when all of the following criteria are met (Abel et al. 2007; Wise \& Abel 2008; Wise et al. 2012):

(1) the gas overdensity of $5 \times 10^{5}\left(\sim 3 \times 10^{3} \mathrm{~cm}^{-3}\right.$ at $z=$ $15)$, 
(2) the metallicity of the gas $<10^{-3.5}$ solar,

(3) the molecular hydrogen fraction $\mathrm{f}_{\mathrm{H}_{2}}<5 \times 10^{-4}$,

(4) the cooling time is less than the dynamical time, $t_{\text {cool }}<$ $t_{\text {dyn }}$,

(5) the velocity flow is converging; i.e., $\nabla \cdot \mathbf{v}<0$.

For the Pop II/I star formation recipe we use the algorithm of Wise et al. (2012) in Enzo. In the simulations, a Pop II/I star particle, representing a stellar cluster, is formed when the following criteria are met: (1) the gas overdensity $5 \times 10^{5},(2)$ the metallicity of the gas $Z>10^{-3.5} Z_{\odot}$, (3) $t_{\text {cool }}<t_{\text {dyn }}$, (4) the velocity flow is converging; i.e., $\nabla \cdot \mathbf{v}<0$. In our simulations, the minimum mass of a Pop II stellar cluster is $10^{3} M_{\odot}$. For every star particle that is created, ionizing radiation transport is included following the prescriptions of Wise et al. (2012), i.e., every star particle radiates UV photons for $20 \mathrm{Myr}$. The energy injection by the supernova explosions ( $\mathrm{SNe}$ ) of Pop III stars is computed from the stellar mass and is deposited in a sphere of $10 \mathrm{pc}$ radius. We follow the metal production by $\mathrm{SNe}$ and take into account the metal cooling.

\subsection{XDR Grids}

We tabulate the XDR grids using a modified version of the chemical network of Meijerink \& Spaans (2005). Our chemical network consists of 176 species and more than 1000 reactions.

The main heating mechanism in XDRs is Coulomb heating when fast electrons interact with thermal electrons, where the heating efficiency is as high $10-50 \%$ (Maloney et al. 1996). Moreover, X-rays have small absorption crosssections, which roughly scale as $1 / E^{3}$, and thus they can penetrate large columns. An important quantity in XDRs that affects the chemo-thermal state of the gas is the energy deposition rate in the gas parcel. In Paper I, we found that the energy deposition rate in a solar metallicity gas is much higher than for the zero metallicity case, causing high temperatures $(\sim$ $10^{6} \mathrm{~K}$ ) in the central $40 \mathrm{pc}$. Furthermore, earlier work on the X-ray effects from an AGN by Pérez-Beaupuits et al. (2011) has shown that X-ray exposed molecular gas has temperatures five times higher than gas in a starburst of equal bolometric power. This has important consequences for the initial mass function (IMF) of stars, because the Jeans Mass $\left(M_{\mathrm{J}}\right)$ scales with the temperature of the ambient gas as $M_{\mathrm{J}} \propto T^{3 / 2}$.

In XDRs at high temperatures $(\mathrm{T}>5000 \mathrm{~K})$, the gas cooling is dominated by collisional excitation of $\mathrm{Ly} \alpha$, and forbidden transitions of [O I] $(\lambda \lambda 6300,6363 \mu \mathrm{m}),[\mathrm{C} \mathrm{I}](\lambda \lambda 9823$, $9850 \mu \mathrm{m}),[\mathrm{Fe} \mathrm{II}](\lambda \lambda 1.26,1.64 \mu \mathrm{m})$, and [Si II] $(\lambda \lambda 6716$, $6731 \mu \mathrm{m})$. At low temperatures $(T<3000 \mathrm{~K})$, gas cooling is dominated by the fine-structure lines of [OI] $63 \mu \mathrm{m}$, [SiII] $35 \mu \mathrm{m},[\mathrm{CII}] 158 \mu \mathrm{m},[\mathrm{CI}] 269$ and $609 \mu \mathrm{m}$, as well as rotational lines of $\mathrm{CO}$ and $\mathrm{H}_{2} \mathrm{O}$. For further details, we refer the interested reader to Meijerink \& Spaans (2005) and Paper I.

We construct and utilize tables for species abundances and gas temperatures over a wide range of X-ray flux $F_{\mathrm{X}}=10^{-1.25}-10^{5.5} \mathrm{erg} \mathrm{cm}^{-2} \mathrm{~s}^{-1}$, number density $n=10-$ $10^{6} \mathrm{~cm}^{-3}$, column density $N_{\mathrm{H}}=10^{20}-10^{24} \mathrm{~cm}^{-2}$, equally spaced with a step-size of $0.25 \mathrm{dex}$, and metallicity $\mathrm{Z} / \mathrm{Z}_{\odot}=$ $10^{-6}, 10^{-4}, 10^{-2}, 1$. This large parameter space enables us to model the interstellar medium (ISM) properties close to an AGN properly. We use Enzo's 9 species $\left(\mathrm{H}, \mathrm{H}^{+}, \mathrm{H}^{-}, \mathrm{He}, \mathrm{He}^{+}\right.$, $\mathrm{He}^{2+}, \mathrm{H}_{2}, \mathrm{H}_{2}^{-}$, and $\mathrm{e}^{-}$) non-equilibrium chemical network for zero metallicity gas (Abel et al. 1997; Anninos et al. 1997) in regions that are not X-ray dominated.

\section{RESULTS \& IMPLICATIONS}

In order to investigate the effects of X-ray irradiation on the growth of MBHs and on the formation and evolution of stellar population in the host halo we perform cosmological, radiation hydrodynamics simulations. The growth of MBHs is simulated under the influence of two different LW background radiation fields: $10^{3}$ and $10^{5} J_{21}$. Here, we assume that there is a close by star-forming halo (Pop II stars with average surface temperatures $T=10^{4} \mathrm{~K}$ ) within $10 \mathrm{kpc}$ which could provide such high LW fluxes (Dijkstra et al. 2008). We insert the seed $\mathrm{MBH}$ with a mass of $M_{\mathrm{BH}}=5 \times 10^{4} M_{\odot}$ into the center of our favorite halo $\left(M_{\mathrm{H}}=2 \times 10^{8} M_{\odot}\right)$ at $z=15$. However, we turn on the radiation field, $\mathrm{H}_{2}$ self-shielding and the star formation modules at $z=30$, before we insert our $\mathrm{MBH}$ to the center of our halo. This is done to check whether the conditions for the DCBH formation scenario are met in our favorite, hosting dark matter halo. We only follow growth of DCBHs for $100 \mathrm{Myr}$, due to high computational expenses of the radiative transfer.

\subsection{High LW Case $\left(B G_{5}=10^{5} J_{21}\right)$}

The only initial difference between the $B G_{5}$ and $B G_{3}$ runs is the strength of the LW background radiation that photodissociates $\mathrm{H}_{2}$ very efficiently. Thus, prior to the DCBH formation at $z=15$, the $\mathrm{H}_{2}$ fraction $\left(f_{\mathrm{H}_{2}}\right)$ in the inner $100 \mathrm{pc}$ of our host halo is as low as $10^{-8}$. This is shown in Figure 1, where we plot gas density, temperature and $\mathrm{H}_{2}$ fraction slices in the y-plane through the densest point at $z=15$ for the $B G_{5}$ (top) and $B G_{3}$ (bottom) runs. The corresponding $\mathrm{H}_{2}$ column density (for a typical density of $10^{-21} \mathrm{~g} \mathrm{~cm}^{-3}$ ) over a scale of $30 \mathrm{pc}$ is $10^{15} \mathrm{~cm}^{-2}\left(f_{s h}=0.17\right)$. Thus, self-shielding occurs but it is not very strong yet, given the broad line wings in the $\mathrm{H}_{2}$ dissociative bands. Under these initial conditions, in our atomic cooling halo with $T_{\text {vir }} \sim 10^{4} \mathrm{~K}$, we therefore do not form any Pop III stars prior to $z=15$. Hence, our DCBH scenario for the formation of the seed $\mathrm{MBH}$ is appropriate.

In the center of our halo, the MBH immediately starts accreting and emitting $\mathrm{X}$-ray radiation. This X-ray radiation increases $e^{-}$fraction which enhances $\mathrm{H}_{2}$ formation in zerometallicity gas through the $\mathrm{H}^{-}$route

$$
\begin{aligned}
H+e^{-} & \rightarrow H^{-}+\gamma, \\
H+H^{-} & \rightarrow H_{2}+e^{-} .
\end{aligned}
$$

In Figure 2, we plot the $\mathrm{H}_{2}$ fraction in the inner $500 \mathrm{pc}$ for both $B G_{5}$ (right) and $B G_{3}$ (left) cases, $\sim 1.3$ Myr after the seed $\mathrm{MBH}$ was inserted. After being irradiated for $\sim 0.5 \mathrm{Myr}$, the $\mathrm{H}_{2}$ abundance increases to $5 \times 10^{-4}$, which is the criterion for Pop III star formation in our simulations, and this nuclear region experiences X-ray induced Pop III star formation. Note that there is gas at $r \sim 20-40 \mathrm{pc}$ with higher $f_{\mathrm{H}_{2}}$ than our Pop III criterion, which is absent in the $B G_{3}$. This is due to the fact that X-rays penetrate further, $\sim 500 \mathrm{pc}$, in the $B G_{5}$ case and thus boost the $f_{\mathrm{H}_{2}}$. In order to check whether this rapid Pop III formation is purely due to the X-ray irradiation from the central $\mathrm{MBH}$ we conducted another simulation with the same setup but without X-ray radiation. In this simulation Pop III stars formed only $\sim 10$ Myrs after we insert the MBH. Hence, we conclude that X-ray irradiation from the central $\mathrm{MBH}$ initially has a positive feedback effect on the star formation, by inducing $\mathrm{H}_{2}$ formation through the $\mathrm{H}^{-}$route, and 

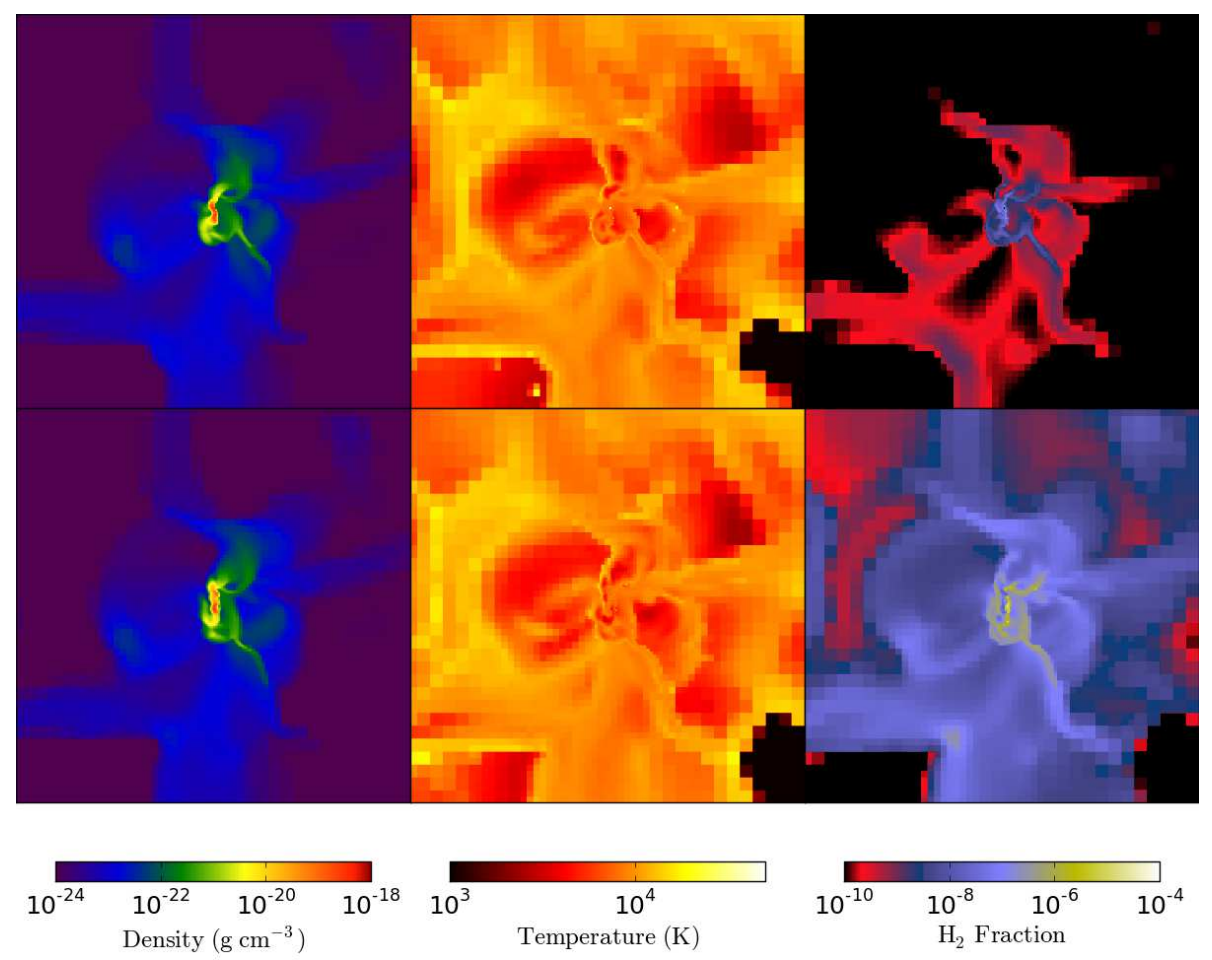

Figure 1. Gas density, temperature and $\mathrm{H}_{2}$ fraction slices in the y-plane at $z=15$ for the $B G_{5}$ (top) and $B G_{3}$ (bottom) runs with a 1 kpc field of view. Prior to the DCBH formation the only difference in between the two simulations is the $\mathrm{H}_{2}$ fraction of the hosting halo.

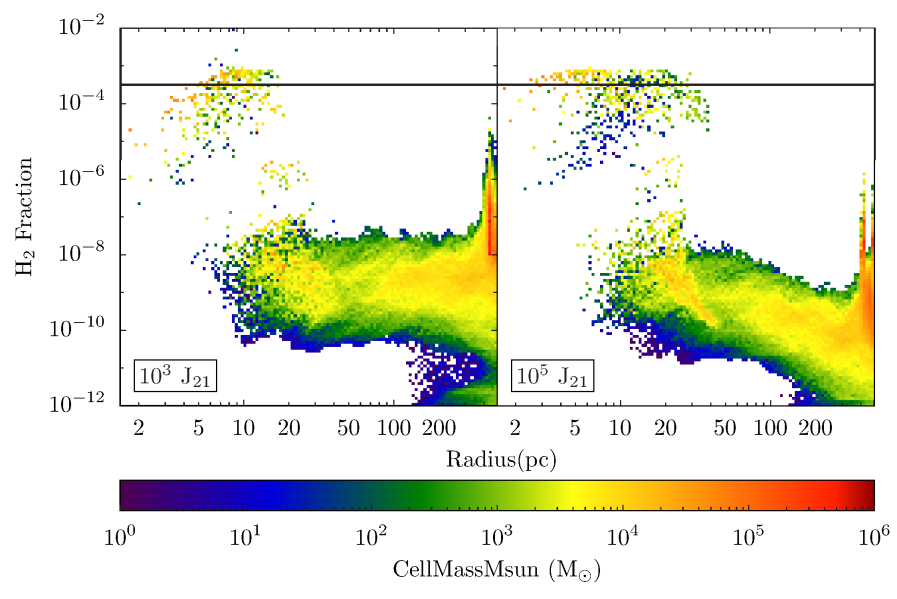

Figure 2. $\mathrm{H}_{2}$ fraction vs. radius for $B G_{3}$ (left) and $B G_{5}$ (right) cases, 1.265 Myrs after the seed $\mathrm{MBH}$ is inserted. The black horizontal line correspond to our Pop III star formation criteria. There is more gas at $\mathrm{r}>10 \mathrm{pc}$ which is above the Pop III star formation threshold in the $B G_{3}$ case. This is due to the fact that X-rays penetrate further, $\sim 500 \mathrm{pc}$, in the $B G_{5}$ case.

thus it is very important to take into account not only for the $\mathrm{MBH}$ growth but also for regulating the stellar population in the host halo.

In Figure 3, we plot metallicity, X-ray flux and $\mathrm{H}_{2}$ fraction slices of $1 \mathrm{kpc}$ through the centre of the $\mathrm{MBH}$ for $B G_{5}$ (top) and $B G_{3}$ (bottom) cases at $z=14.86$ (3.6 Myr after the $\mathrm{MBH}$ is inserted), when Pop III SNe first occur in the halo. Due to the strong LW background, the $\mathrm{H}_{2}$ abundance and thus cooling in the halo is efficiently suppressed, leading to high temperatures and low densities. Thus, the strong LW background renders the host halo fragile to radiative feedback both from $\mathrm{SNe}$ and the $\mathrm{MBH}$ and thus the collective effect on the ambient gas is hostile. As illustrated in Figure 3, the SNe blast-waves heat and destroy any $\mathrm{H}_{2}$ in their wake and subsequently chemically enriches the surrounding medium. X-ray radiation penetrates up to $500 \mathrm{pc}$ from the center, due to the very strong LW background leading low column densities and optical depth for X-rays. When the SNe go off, they form HII regions which propagate much faster in the BG5 case. This is because for a local ionizing photon rate $S_{i}$, the Strömgren radius scales as $R_{S} \propto\left(S_{i} / n^{2}\right)^{1 / 3}$, the recombination time scales as $t_{s} \propto 1 / n_{e}$, and the ionization front velocity scales as $V_{I} \propto S_{i} / n \times R^{2}$. This in turn, provides a path for metals to enrich the ISM up to $\sim 1$ kpc.

In Figure 4, we plot temperature versus radius color-coded by metallicity (left panel) and X-ray flux (right panel) for both simulations. The $\mathrm{SNe}$ blast-waves propagate faster in the $B G_{5}$ case and blow away the gas from the inner $\sim 15 \mathrm{pc}$. Although the X-ray irradiation from the $\mathrm{MBH}$ is weak, due to the lack of gas, it penetrates to larger distances from the $\mathrm{MBH}$ with respect to the $B G_{3}$ case (right panel of Figure 4).

Figure 5 shows the accretion rate (solid) and corresponding total energy production (dashed) for both $B G_{3}$ (left) and $B G_{5}$ (right) runs for $100 \mathrm{Myr}$. The MBHs initially accrete gas efficiently at a rate $\sim 10^{-3} M_{\odot} \mathrm{yr}^{-1}$ (10\% Edddington rate), 2 Myr after they are inserted into the simulation. This accretion rate corresponds to a total energy production of $2 \times 10^{56}$ erg over 1 Myr at radiative efficiency $\epsilon=0.1$, which is about the same as the energy produced by $2 \times 10^{5}$ SNe explosions. The red-colored part in the right-panel of Figure 5 shows the hot gas $\left(T \geq 10^{4} \mathrm{~K}\right)$ dominated accretion rate whereas the blue-colored part shows the cold gas $T<10^{4} \mathrm{~K}$ ) dominated accretion rate. This implies that sound speed $\left(c_{s}^{3}\right)$ in the equation 3 is more strongly dependent on the temperature of the gas $\left(\propto T^{-3 / 2}\right)$ than the density of the gas $\left(\rho_{B}\right)$. Overall gas densities in the inner 30 pc are 2-3 orders of magnitude lower $\left(\rho \sim 10^{-23} \mathrm{~g} \mathrm{~cm}^{-3}\right)$ than the $B G_{3}$ case. Thus, the response time of the ambient gas to both UV and $\mathrm{X}$-ray radiation is 


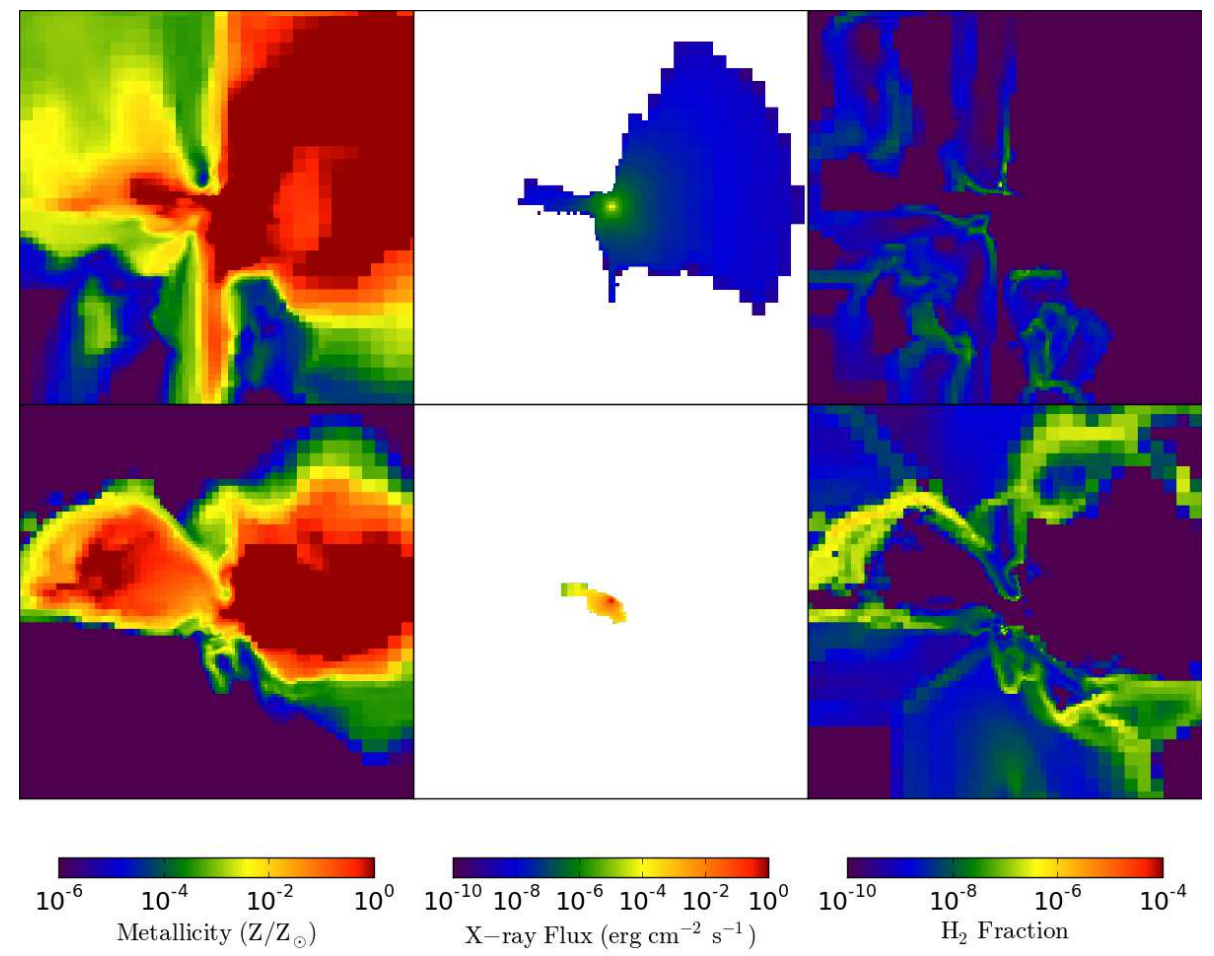

Figure 3. Metallicity, X-ray flux and $\mathrm{H}_{2}$ fraction slices in the $\mathrm{y}$-plane with a $1 \mathrm{kpc}$ field of view through the centre of the $\mathrm{MBH}$ for $B G_{5}$ (top) and $B G_{3}$ (bottom) runs at $z=14.86$ when Pop III SNe first occur in the halo(3.6 Myrs after the MBH is inserted). In the $B G_{5}$ case $\mathrm{X}$-rays penetrate further, $\sim 500$ pc.

much longer. This also helps to explain the longer duty cycles seen in $B G_{5}$ run and we discuss this further in Section 3.2.

In order to investigate the dependence of the MBH growth on the local versus large scale gas thermodynamics we perform a simulation where we insert the MBH $10 \mathrm{pc}$ off the center of the potential well of the host halo. In this case, because the $\mathrm{MBH}$ does not reside in the most dense region in the halo, the enhancement in the $\mathrm{H}_{2}$ fraction by the X-ray irradiation was not enough to instigate an $\mathrm{H}_{2}$-driven collapse. This is very important because without star formation there is no metal enrichment.

Indeed, in this simulation setup, the enhancement of $\mathrm{H}_{2}$ from the $\mathrm{X}$-ray radiation (through $\mathrm{H}^{-}$route) was not high enough to induce a collapse, keeping the halo star-free and thus metal-free for $100 \mathrm{Myr}$. The response of metal-free gas to the high $\mathrm{LW}$ radiation, which dissociates $\mathrm{H}_{2}$ and thus keep the gas hot and diffuse, was so long that the accretion rate was 4 orders of magnitude less than the original $B G_{5}$ case. Thus, the $\mathrm{MBH}$ grew only $1 \%$ in $100 \mathrm{Myr}$ whereas in the original run it grew $\sim 6 \%$ as shown in Figure 6 .

On the other hand, the duty cycle of the two runs are not so different. We derived the duty cycle of these to simulations to be $4 \%$ and $6 \%$ for off-center and original run, respectively. Therefore, we conclude that the maximum accretion rate is determined by the local gas thermodynamics whereas the duty cycle is determined by the large scale gas dynamics and gas reservoir of the host halo. However, even in the original $B G_{5}$ case, the $\mathrm{MBH}$ grows only at $10 \%$ Eddington rate. This is shown in Figure 6, where we plot the growth of $\mathrm{MBH}$ over $100 \mathrm{Myr}$ for $B G_{5}$ (red-dashed line) and $B G_{3}$ (black-solid line) cases.

\subsection{Low $L W$ case $\left(B G_{3}=10^{3} J_{21}\right)$}

In the $B G_{3}$ case, prior to $z=15$, the $\mathrm{H}_{2}$ abundance fraction is $\sim 10^{-6}$ (100 times higher than the $B G_{5}$ case $)$, as shown in Figure 1. Recent works by Inayoshi \& Omukai (2012) and Visbal et al. (2014), have shown that in order to form a DCBH a zone of no-return should be reached before runaway cooling begins. This requires not only low $f_{\mathrm{H}_{2}}\left(\leq 10^{-6}\right)$, but also high initial densities $\left(n>10^{4} \mathrm{~cm}^{-3}\right)$ and temperatures $\left(T \geq 10^{4}\right.$ $\mathrm{K})$. Since, the $B G_{3}$ simulation prior to $z=15$ meets these criteria ( $n \sim 10^{6} \mathrm{~cm}^{-3}$ and $T>10^{4} \mathrm{~K}$, see Figure 1$)$, our DCBH scenario still holds.

After 0.5 Myr, the X-ray radiation from the $\mathrm{MBH}$ enhances the $\mathrm{H}_{2}$ abundance to $>5 \times 10^{-4}$, instigating a collapse and fragmentation forming Pop III stars. Metal enrichment by Pop III stars provides a high opacity for X-rays because absorptions by inner shell electrons of $\mathrm{C}, \mathrm{N}$ and $\mathrm{O}$ have large cross sections above $1 \mathrm{keV}$. In Figure 4 (right panel), the Xray flux is strongly attenuated by the ISM in the inner $20 \mathrm{pc}$. $\mathrm{X}$-rays do not penetrate above $30 \mathrm{pc}$ due to the high column densities, on the order of $10^{23-24} \mathrm{~cm}^{-2}$, that are reached in the center. Therefore, the energy deposition rate into the ISM is higher than in the $B G_{5}$ case (also see Paper I). Furthermore, as studied in Paper I, due to the large cross-sections available to $\mathrm{X}$-rays penetrating metal-rich gas, the temperature of the high density high-metallicity gas is higher than the low-metallicity gas, also shown in Figure 4. This is converse of the normal behavior of metals in an X-ray free environment. Under normal conditions, where there are no X-rays, metals are responsible for cooling of the gas. However, when there are X-rays, the metal-rich gas is heated due to the high cross-sections. Therefore, it is very important to take into account X-ray feedback effects when studying BH growth where the ISM is enriched by the metals.

The accretion rate after $1 \mathrm{Myr}$ is $10^{-3} M_{\odot} \mathrm{yr}^{-1}$ (see Figure 5), similar to the $B G_{5}$ case. Afterwards the $\mathrm{MBH}$ accretes at this high rate more frequently than the $B G_{5}$ case because the nuclear gas can cool more efficiently through $\mathrm{H}_{2}$ due to 

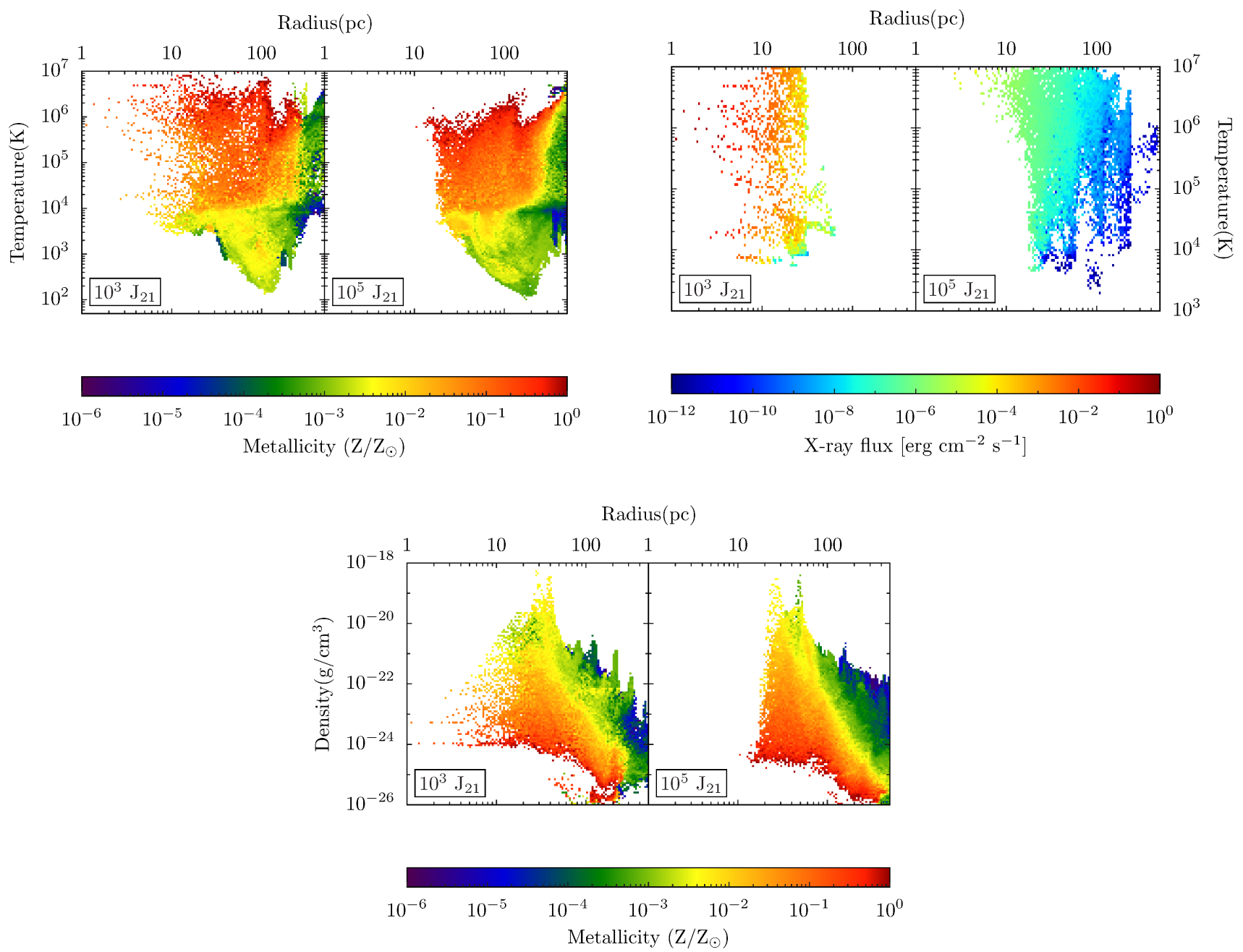

Figure 4. Top panel: temperature versus radius color-coded by metallicity (Ieft panel) and X-ray flux (right panel) for both $B G_{5}$ and $B G_{3}$ simulations at $z=14.86$ (3.6 Myr after the MBH is inserted). The SNe blast-waves propagate faster in the $B G_{5}$ case and blow away the gas from the inner $\sim 15$ pc. Bottom panel: density versus radius color-coded by metallicity. The temperature of the high density high-metallicity gas is higher than the low-metallicity gas due to the large cross-sections available to X-rays penetrating metal-rich gas.

the lower LW background. In fact, for the $B G_{3}$ run we obtain a duty cycle of close to $50 \%$. The $B G_{5}$ case yields an order of magnitude less $(6 \%)$. These numbers are similar to Park \& Ricotti (2012). In their terminology, the $B G_{5}$ and $B G_{3}$ runs experience "mode-I" (6\%) and "mode-II" (50\%) accretion. On the other hand, the $\mathrm{MBH}$ in the $B G_{3}$ case does not double its mass in an Eddington time (45 Myr) either. As shown in Figure 6, it grows only by $16 \%$ in 100 Myr. We conclude that, once radiative feedback from the accreting $\mathrm{MBH}$ is taken into account, under the influence of an external LW background, the $\mathrm{MBH}$ growth is stifled relative to the typical Eddington rate argument.

\section{DISCUSSION \& CONCLUSIONS}

In this work, we investigate the role of X-ray radiation on regulating $\mathrm{MBH}$ growth and facilitating stellar populations in the host dark matter halo, under the influence of a LW background. Here we summarize the interplay between the physics we included in the simulations. The LW background dissociates and suppresses $\mathrm{H}_{2}$ formation. Once the seed DCBH forms, it starts accreting and producing $\mathrm{X}$-rays. This radiation increases the $e^{-}$abundance which induces $\mathrm{H}_{2}$ formation in the primordial gas. Then Pop III stars form in our simula- tions when cold, $\mathrm{H}_{2}$ rich, and dense clouds form. Their $\mathrm{SNe}$ enrich the ISM, allowing gas to cool as usual in the absence of strong X-ray radiation. When the seed DCBH accretes gas it produces $\mathrm{X}$-rays that are attenuated by metal-rich gas causing efficient heating. There is thus an interplay between X-rays and the LW background depending on ambient metallicity and the resulting duty cycle of $\mathrm{BH}$ accretion. The highlights of our findings are as follows.

- The presence of a strong LW background renders a primordial atomic cooling halo of $\sim 10^{8} M_{\odot}$ fragile to radiative feedback by $\mathrm{SNe}$ and a $\mathrm{MBH}$.

- The X-ray irradiation from the central MBH induces the initial star formation and Pop III stars form 0.5 Myr after the seed MBH was inserted. However, in the long term it prevents the $\mathrm{MBH}$ from accreting at high rates continuously.

- The X-ray feedback and MBH growth is selfregulating. The maximum accretion rate that both MBHs experience is similar, $10^{-3} M_{\odot} \mathrm{yr}^{-1}$. On the other hand, the duty cycle of these two MBHs is derived to be $6 \%$ and $50 \%$ for high and low LW cases, respectively. 

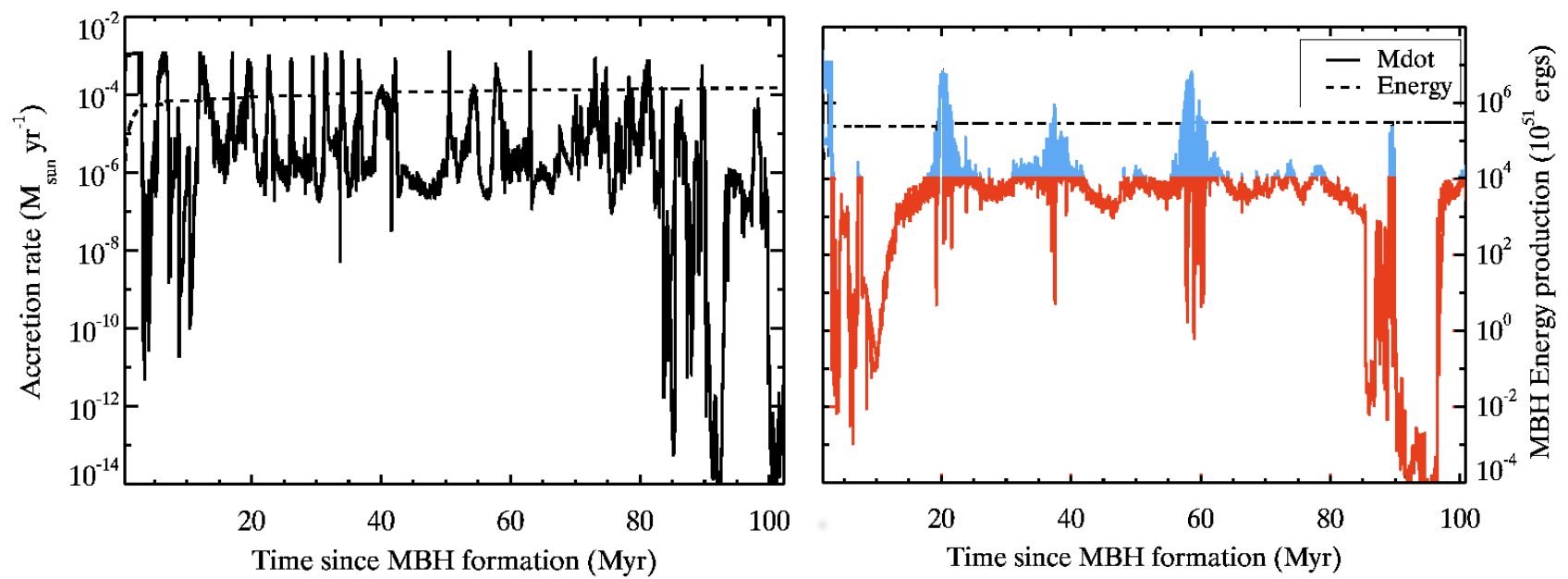

Figure 5. Accretion rate and the corresponding total energy production of the central $\mathrm{MBH}$ over time for the $B G_{3}$ (left) and $B G_{5}$ (right) runs. The maximum accretion rate that both MBHs experience is similar, $10^{-3} \mathrm{M}_{\odot} \mathrm{yr}^{-1}$. However, the duty cycle of these two MBHs is derived to be $6 \%$ and $50 \%$ for the $B G_{5}$ and $B G_{3}$ cases, respectively. The red-colored part on the $B G_{5}$ plot shows the hot gas $\left(T \geq 10^{4} \mathrm{~K}\right)$ dominated accretion whereas the blue-colored part shows the cold gas $\left(T<10^{4} \mathrm{~K}\right)$ dominated accretion.

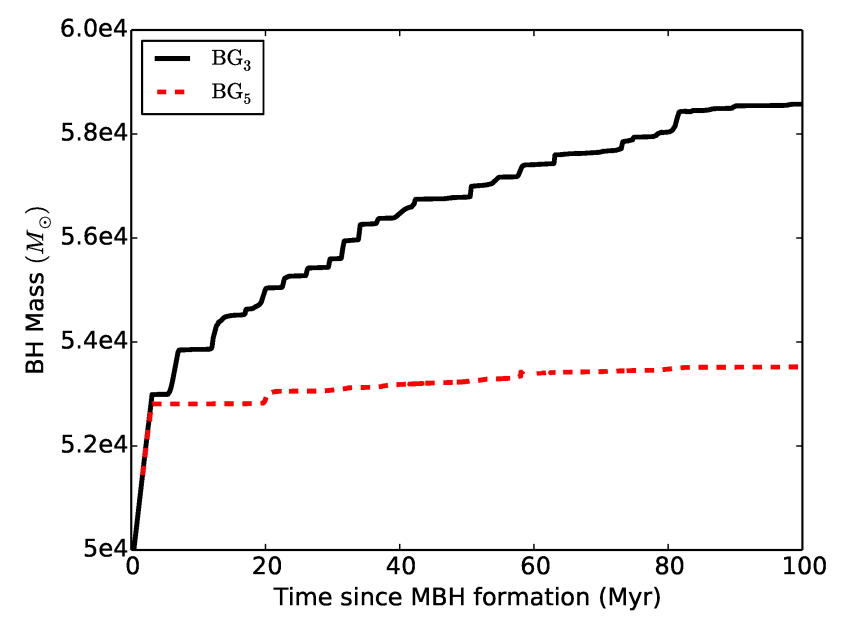

Figure 6. Growth of the MBH over $100 \mathrm{Myr}$ for the $B G_{5}$ (red-dashed line) and $B G_{3}$ (black-solid line) cases. In either cases, the $\mathrm{MBH}$ growth is stifled relative to the typical Eddington rate argument.

The MBH in the high LW case grows only $\sim 6 \%$ in $100 \mathrm{Myr}$ whereas in the low LW case the MBH grows $16 \%$ in $100 \mathrm{Myr}$. We find that this is due to the fact that the maximum of the accretion rate is determined by the local gas thermodynamics whereas the duty cycle is determined by the large scale gas dynamics and gas reservoir of the host halo.

- The initial chemical enrichment is very crucial for MBH growth. Metal rich gas has shorter response times to X-ray radiative feedback which enables gas to accrete onto the $\mathrm{MBH}$.

- Once X-ray feedback effect from the accreting MBHs is taken into account, under the influence of external LW background radiation, the MBH growth is stifled relative to the typical Eddington rate argument. Even if the DCBH starts accreting at the Eddington rate af- ter a few Eddington times and can maintain this accretion through cosmic time, then the resulting mass gain would still be a factor of $\sim e^{2}$ less.

We here stress that, $N_{\mathrm{H}_{2}}$ is a non-local quantity, but because of the high computational expenses of determining selfshielding accurately, we have estimated the $\mathrm{H}_{2}$ self-shielding effect by using a local approximation. This local approximation method has been shown to be accurate within an order of magnitude only (Shang et al. 2010; Wolcott-Green et al. 2011). Wolcott-Green et al. (2011) have shown that the Sobolev length method is more accurate than the Jeans length to estimate the $N_{\mathrm{H}_{2}}$, especially for densities $n<10^{4} \mathrm{~cm}^{-3}$, which reduces the critical specific intensity needed to keep halos $\mathrm{H}_{2}$ free by a factor of six. In our simulations, gas reaches densities of $n=10^{6} \mathrm{~cm}^{-3}$ within the inner $40 \mathrm{pc}$, and we considered $J_{21}$ values that are above the critical value, above which the halo remain $\mathrm{H}_{2}$-free. Hence, in the scenarios presented here, considering the Sobolev length would not change our conclusions on the formation of MBH. However, it might affect the stellar population in the host halo.

Our simulations assume a constant LW background, which might cause the lower accretion rates since the source of the LW radiation field and its effect on our halo depends on the position and evolution of the source (Regan et al. 2014).

The spatial resolution of our simulations is $3.6 \mathrm{pc}$ hence, everything within $\sim 4 \mathrm{pc}$ is accreted by the MBH. This might lead us to overestimate the accretion rates. However, the accretion prescription we use in this work, Eddington limited spherical Bondi-Hoyle, does not include the angular momentum of the gas which is crucial in order for gas to fall onto the MBH from the accretion disk. In fact, Power et al. (2011) showed that taking into account the angular momentum of the accreting gas can influence the response of the ambient gas to the feedback effect from the MBH. Therefore, in the near future, we are planning to perform higher-resolution simulations with a better accretion prescription that will take into account the angular momentum of the infalling gas with an evolving LW background. 
We would like to thank the referee for their comments which made the overall paper clearer. This research was supported by National Science Foundation (NSF) grants AST-1211626 and AST-1333360. AA is grateful to Andrea Ferrara for stimulating discussions on black hole growth. Computations and associated analysis described in this work were performed using the publiclyavailable Enzo code (http://enzo-project.org) and the $y t$ toolkit (http://yt-project.org; Turk et al. 2011).

\section{REFERENCES}

Abel, T., Anninos, P., Zhang, Y., \& Norman, M. L. 1997, New A, 2, 181 Abel, T., Wise, J. H., \& Bryan, G. L. 2007, ApJ, 659, L87

Agarwal, B., Davis, A. J., Khochfar, S., Natarajan, P., \& Dunlop, J. S. 2013, MNRAS, 432, 3438

Agarwal, B., Khochfar, S., Johnson, J. L., Neistein, E., Dalla Vecchia, C., \& Livio, M. 2012, MNRAS, 425, 2854

Anninos, P., Zhang, Y., Abel, T., \& Norman, M. L. 1997, New A, 2, 209

Aykutalp, A., Wise, J. H., Meijerink, R., \& Spaans, M. 2013, ApJ, 771, 50

Begelman, M. C., \& Rees, M. J. 1978, MNRAS, 185, 847

Bromm, V., \& Loeb, A. 2003, ApJ, 596, 34

Bryan, G. L., et al. 2014, ApJS, 211, 19

Dijkstra, M., Ferrara, A., \& Mesinger, A. 2014, MNRAS, 442, 2036

Dijkstra, M., Haiman, Z., Mesinger, A., \& Wyithe, J. S. B. 2008, MNRAS, 391, 1961

Draine, B. T., \& Bertoldi, F. 1996, ApJ, 468, 269

Ebisuzaki, T., et al. 2001, ApJ, 562, L19

Fan, X., et al. 2003, AJ, 125, 1649

-. 2006, AJ, 131, 1203
Haehnelt, M. G., \& Rees, M. J. 1993, MNRAS, 263, 168

Inayoshi, K., \& Omukai, K. 2012, MNRAS, 422, 2539

Johnson, J. L., \& Bromm, V. 2007, MNRAS, 374, 1557

Kim, J.-h., Wise, J. H., Alvarez, M. A., \& Abel, T. 2011, ApJ, 738, 54

Kurk, J. D., et al. 2007, ApJ, 669, 32

Latif, M. A., Bovino, S., Van Borm, C., Grassi, T., Schleicher, D. R. G., \& Spaans, M. 2014a, MNRAS, 443, 1979

Latif, M. A., Schleicher, D. R. G., Bovino, S., Grassi, T., \& Spaans, M. 2014b, ArXiv e-prints

Maloney, P. R., Hollenbach, D. J., \& Tielens, A. G. G. M. 1996, ApJ, 466, 561

Meijerink, R., \& Spaans, M. 2005, A\&A, 436, 397

Mellema, G., Iliev, I. T., Alvarez, M. A., \& Shapiro, P. R. 2006, New A, 11, 374

Mortlock, D. J., et al. 2011, Nature, 474, 616

O'Shea, B. W., \& Norman, M. L. 2008, ApJ, 673, 14

Park, K., \& Ricotti, M. 2012, ApJ, 747, 9

Pérez-Beaupuits, J. P., Wada, K., \& Spaans, M. 2011, ApJ, 730, 48

Power, C., Nayakshin, S., \& King, A. 2011, MNRAS, 412, 269

Regan, J. A., Johansson, P. H., \& Wise, J. H. 2014, ArXiv e-prints

Shang, C. Bryan, G. L. \& Haiman, Z 2010, MNRAS, 402, 1249

Sobolev, V. V. 1957, Soviet Ast., 1, 678

Spaans, M., \& Silk, J. 2006, ApJ, 652, 902

Turk, M. J., Smith, B. D., Oishi, J. S., Skory, S., Skillman, S. W., Abel, T., \& Norman, M. L. 2011, ApJS, 192, 9

Umemura, M., Loeb, A., \& Turner, E. L. 1993, ApJ, 419, 459

Visbal, E., Haiman, Z., \& Bryan, G. L. 2014, MNRAS, 442, L100

Volonteri, M., Haardt, F., \& Madau, P. 2003, ApJ, 582, 559

Volonteri, M., \& Rees, M. J. 2005, ApJ, 633, 624

Wise, J. H., \& Abel, T. 2008, ApJ, 685, 40

-. 2011, MNRAS, 414, 3458

Wise, J. H., Turk, M. J., \& Abel, T. 2008, ApJ, 682, 745

Wise, J. H., Turk, M. J., Norman, M. L., \& Abel, T. 2012, ApJ, 745, 50

Wolcott-Green, J., Haiman, Z., \& Bryan, G. L. 2011, MNRAS, 418, 838 